\title{
ESTUDO DA REGENERAÇÃO NATURAL DE ESPÉCIES ARBÓREAS EM FRAGMENTO DE FLORESTA OMBRÓFILA DENSA, MATA DAS GALINHAS, NO MUNICÍPIO DE CATENDE, ZONA DA MATA SUL DE PERNAMBUCO
}

\author{
NATURAL REGENERATION'S STUDY OF ARBOREAL SPECIES IN MATA DAS GALINHAS \\ FRAGMENT, HUMID FOREST IN CATENDE'S CITY, PERNAMBUCO'S SOUTH ZONE
}

\author{
Wegliane Campelo da Silva ${ }^{1}$ Luiz Carlos Marangon ${ }^{2}$ Rinaldo Luiz Caraciolo Ferreira ${ }^{3}$ \\ Ana Lícia Patriota Feliciano ${ }^{4}$ Roberto Felix Costa Junior ${ }^{5}$
}

\section{RESUMO}

O estudo foi desenvolvido no fragmento denominado Mata das Galinhas, situado no município de Catende - PE, na mesorregião da mata pernambucana, mais precisamente na microrregião da mata úmida, a 142 Km da capital, nas coordenadas 869’06”S e 3569’08” W, com altitude média de 199m. O relevo varia, predominantemente, de ondulado a forte ondulado. Os solos predominantes são classificados como latossolo vermelho distrófico e nitossolo vermelho associado ao latossolo. A vegetação é de Floresta Ombrófila Densa. Neste estudo, objetivou-se avaliar a regeneração natural de espécies arbóreas no fragmento florestal e verificar a diversidade e a estrutura das espécies no mesmo. Para a estimativa da regeneração natural das espécies arbóreas, foram locadas, de forma sistemática, 16 subparcelas de $25 \mathrm{~m}^{2}$ ( 5 x $5 \mathrm{~m}$ ), no centro de 16 unidades amostrais permanentes de $250 \mathrm{~m}^{2}$ (10,0 x 25,0 m), para o estudo da fitossociologia da comunidade arbórea adulta, com um distanciamento de $50 \mathrm{~m}$ entre si. O nível de inclusão foi de CAP $\leq 15 \mathrm{~cm}$, e a medição de altura (h) foi dividida em classes, em que a classe 1 contemplou indivíduos com $1,0 \leq \mathrm{h} \leq 2,0 \mathrm{~m}$, a classe 2 com indivíduos 2,0 $<\mathrm{h} \leq 3,0 \mathrm{~m}$ e a classe, 3 indivíduos com $\mathrm{h}>3,0 \mathrm{~m}$. Foram realizados os cálculos da suficiência amostral e da diversidade. No fragmento, foram amostrados 194 indivíduos, pertencentes a 31 famílias botânicas e a 60 espécies arbóreas. Destas, 42 foram identificadas em nível de espécie; 4,em nível de gênero; 2, em nível de família; e 2, indeterminadas. As dez espécies com maiores valores para regeneração natural Total da População Amostrada (RNT) representaram 53,78\%, estando assim distribuídas: Brosimum discolor Schott (9,98\%), Protium heptaphyllum (Aubl.) Marchand (9,19\%), Eschweilera ovata (Cambess.) Miers (8,01\%), Thyrsodium spruceanum Benth. (7,0\%), Dialium guianense (Aubl.) Sandwith (5,44\%), Erythroxylum squamatum Sw. (3,32\%), Cupania revoluta Rolfe (3,16\%), Sorocea hilarii Gaudich. (2,66\%), Cordia nodosa Lam. (2,52\%) e Tapirira guianensis Aubl. (2,50\%). Tais espécies apresentaram capacidade de regeneração no fragmento, sendo que, no futuro, provavelmente, serão as principais responsáveis pela manutenção da estrutura e fisionomia da floresta. O índice de diversidade encontrado para o fragmento foi de 3,57 nats/ind.

Palavras-chave: regeneração natural; fitossociologia; Floresta Ombrófila Densa; fragmento.

\section{ABSTRACT}

The study was developed in the fragment situated in the Catende's - PE city (Mata das Galinhas $8^{\circ} 69^{\prime} 06^{\prime \prime}$ S; 3569'08" W), in mesoregion of the pernambucana forest, more necessarily in the humid forest's microregion, at $142 \mathrm{~km}$ of the capital. The area's vegetacion is Humid Forest. The relief varies wavy to very wavy, with $199 \mathrm{~m}$ altitude. The soil is classified as red latisols dystrophic and red nitosols associated to latisols. The present work aims to study and evaluated the total natural regeneration of arboreal species in the forest fragment and to verify the diversity and structure of the species in the same one. For estimate the

1. Engenheira Florestal, MSc., Departamento de Ciência Florestal, Universidade Federal Rural de Pernambuco, Rua Araripina, 136, A. Lundgren 1, CEP 53415-130, Paulista (PE). wellcampelo@yahoo.com.br

2. Engenheiro Florestal, Dr., Professor do Departamento de Ciência Florestal, Universidade Federal Rural de Pernambuco, Av. Dom Manoel de Medeiros, s/n, Bairro Dois Irmãos, CEP 52171-900, Recife (PE). marangon@ufrpe.br

3. Engenheiro Florestal, Dr., Professor do Departamento de Ciência Florestal, Universidade Federal Rural de Pernambuco, Av. Dom Manoel de Medeiros, s/n, Bairro Dois Irmãos, CEP 52171-900, Recife (PE). rinaldo@dcfl.ufrpe.br

4. Engenheira Florestal, Dra ${ }^{\text {. }}$, Professor do Departamento de Ciência Florestal, Universidade Federal Rural de Pernambuco, Av. Dom Manoel de Medeiros, s/n, Bairro Dois Irmãos, CEP 52171-900, Recife (PE). licia@ufrpe.br

5. Biológo, MSc., Departamento de Ciência Florestal, Universidade Federal Rural de Pernambuco, Rua Dr. Orlando Cabral, 27, Bairro Centro, CEP 54080-300, Jaboatão dos Guararapes (PE).rfflorestal@yahoo.com.br

Recebido para publicação em 5/06/2006 e aceito em 28/04/2007. 
natural regeneration, were made systematically 16 subunits (samples with $5 \times 5 \mathrm{~m}$ ). These subunits were inserted in the middle samples $(10 \times 25 \mathrm{~m})$ before used to evaluate the community arboreal phytossociology, with 50 meters between the ones. The regeneration study (CAP $\leq 15 \mathrm{~cm}$ ) was developed with heights divided in three categories $(1$ - individuals with minimum heights $1 \leq 2 \mathrm{~m} ; 2$ - individuals with minimum heights $2 \leq$ $3 \mathrm{~m} ; 3$ - individuals with heights $>3 \mathrm{~m}$ ). Besides, the samply sufficient and diversity were calculated. In fragment was observed 31 botanical families and 60 arboreal species inside 194 individuals. 42 had been identified in level of species, 4 in sort level, 2 in family level and 2 are not identify. Ten species obtained bigest values for total natural regeneration of the samples population (RNT) and contribute with 53,78\% of the total percentage, how is descript following way: Brosimum discolor Schott (9,98\%), Protium heptaphyllum (Aubl.) Marchand (9,19\%), Eschweilera ovata (Cambess.) Miers (8,01\%), Thyrsodium spruceanum Benth. (7,0\%), Dialium guianense (Aubl.) Sandwith (5,44\%), Erythroxylum squamatum Sw. (3,32\%), Cupania revoluta Rolfe (3,16\%), Sorocea hilarii Gaudich. (2,66\%), Cordia nodosa Lam. (2,52\%) e Tapirira guianensis Aubl. (2,50\%). These species had presented a good capacity of regeneration, probably they will be main responsible for the maintenance of forest's structure and physiognomy. The diversity $(\mathrm{H}$ ') was 3,65 nats/ind for the fragment.

Keywords: natural regeneration; phytossociology; Humid Forest; fragment.

\section{INTRODUÇÃO}

A ciência florestal está associada a uma elevada quantidade de informações sobre os mais diversos tipos de estruturas e mecanismos biológicos e se preocupa com a constante redução da diversidade natural das áreas verdes sem um manejo adequado. Neste contexto, buscando fundamentar ações voltadas para a conservação dos ecossistemas florestais, enfatiza a necessidade do conhecimento para orientar as decisões de sustentabilidade, em que a intervenção humana não provoque uma profunda perturbação do biótopo natural, podendo ainda, causar mudanças evolutivas e, conseqüentemente, irreversíveis nas inter-relações entre os seres vivos e o meio.

Muitos remanescentes florestais encontram-se na forma de fragmentos altamente perturbados, isolados, pouco conhecidos, desprotegidos e em áreas de encostas. Portanto, ainda há necessidade de se buscar informações para a caracterização de florestas, cujo desenvolvimento ocorra sob influência das pressões diretas do entorno, tais como introdução de monoculturas, pastejo, incêndios freqüentes e corte seletivo de madeira para diversos fins (SANTANA et al., 2004).

No Brasil, a formação das florestas secundárias é atribuída à expansão da fronteira agrícola, aos projetos de urbanização e industrialização e à mineração. Também é resultante da exploração seletiva de madeiras e do corte raso para a agricultura itinerante, o que causa a abertura de grandes clareiras e o surgimento da vegetação secundária (RONDON NETO et al., 2000).

A regeneração natural é uma fase importante para a sobrevivência, desenvolvimento e manutenção do ecossistema florestal (FINOL, 1971), pois representa o conjunto de indivíduos capazes de serem recrutados para os estágios posteriores. Portanto, o estudo da regeneração das florestas constitui-se num tema de relevância para a preservação, conservação e recuperação das florestas.

O dossel atual de uma floresta é composto por árvores que provavelmente iniciaram seu ciclo vital muitos anos atrás e que mantêm, abaixo de si, os sobreviventes de sucessivos períodos de regeneração a partir de sementes ou de outros mecanismos (SWAINE e HALL, 1988), tais como rebrota de cepas ou banco de plântulas. Estes estudos, freqüentemente subestimados, têm grande importância, já que permitem conhecer o desenvolvimento das várias espécies e como elas podem ocupar o estrato arbóreo (BARREIRA et al., 2002).

Desse modo, a regeneração natural permite uma análise efetiva para diagnosticar o estado de conservação do fragmento e a resposta às perturbações naturais ou antrópicas, uma vez que representa o conjunto de indivíduos capazes de serem recrutados para os estádios posteriores.

O fragmento Mata das Galinhas foi escolhido para compor o presente trabalho por estar inserido no município de Catende, uma das poucas regiões estudadas da Floresta Ombrófila Densa, que se encontra situada na Zona da Mata Sul pernambucana, por obter uma das maiores áreas entre os remanescentes locais e também por apresentar melhor acessibilidade. Este trabalho visa estudar e verificar o comportamento das 
espécies florestais arbóreas que se encontram em fase de regeneração no fragmento Mata das Galinhas, com o objetivo de subsidiar informações de conhecimento florístico, fitossociológico e do estado de regeneração da área.

\section{MATERIAL E MÉTODOS}

\section{Caracterização da área}

\section{Localização}

O estudo foi desenvolvido no fragmento denominado Mata das Galinhas, pertencente ao complexo da Usina Catende, que é composta por 48 engenhos, totalizando 26.400 ha. O município de Catende está situado na mesorregião da mata pernambucana, mais precisamente na microrregião da mata úmida, a $142 \mathrm{Km}$ da capital, nas coordenadas $8^{\circ} 40^{\prime} 00^{\prime \prime} \mathrm{S}$ e $35^{\circ} 35^{\prime} 00^{\prime}$ ' W. A altitude da cidade é de $100 \mathrm{~m}$. A temperatura anual varia entre 22 e $26^{\circ} \mathrm{C}$, e a precipitação média anual é de $1.414 \mathrm{~mm}$. O relevo varia de ondulado a fortemente ondulado. O município está inserido na bacia hidrográfica do rio Uma (CONDEPE, 1987).

\section{Fragmento Mata das Galinhas}

A Mata das Galinhas é um fragmento florestal que possui 30,95 ha (Figura 1), está inserido no Engenho Tabaiaré, cujas coordenadas geográficas são 869’06” S e 3569’08” W, apresenta uma elevação de 199 m e uma paisagem fortemente marcada pela monocultura da cana-de-açúcar, a qual circunda todo o fragmento.

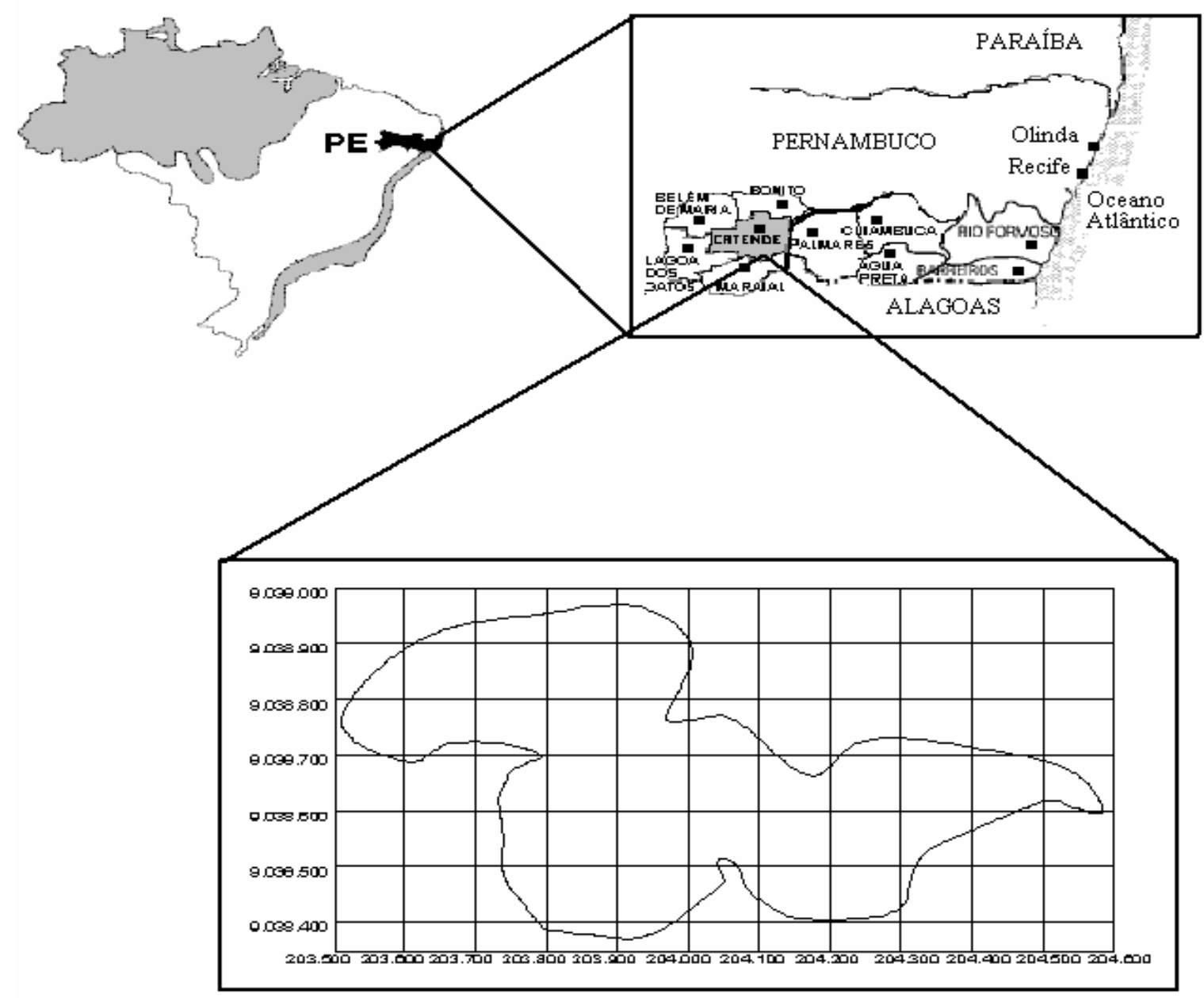

FIGURA 1: Localização do fragmento Mata das Galinhas, Catende - Pernambuco/Brasil, em escala de 1:3500. 2005.

FIGURE 1: Localization of Mata das Galinhas fragment, Catende’s city - Pernambuco/Brazil, 1:3500 scale. 2005. 


\section{Solos}

Os solos predominantes na área geográfica do município são classificados como latossolo vermelho distrófico e nitossolo vermelho associado ao latossolo (EMBRAPA, 2005), os quais são, em geral, solos pobres de nutrientes, de textura argilosa, podendo conter rochas granitizadas.

\section{Vegetação}

Segundo informações obtidas por meio do CONDEPE (1987), a vegetação original remanescente corresponde à formação vegetal representada pela Floresta Estacional Semidecidual, que é uma formação densa, alta (20-30m) e rica em espécies.

Estudando fragmentos da Floresta Atlântica brasileira em locais muito próximos ao município de Catende, Ranta et al. (1998) descreveram as áreas pertencentes às cidades de Ipojuca, Escada, Primavera, Ribeirão, Sirinhaem, Gameleira, Rio Formoso e Barreiros como sendo de formação vegetacional de Floresta Ombrófila Densa.

\section{Análise da Estrutura da Regeneração Natural}

Para a estimativa da regeneração natural das espécies arbóreas, foram locadas, de forma sistemática, 16 subparcelas de $25 \mathrm{~m}^{2}$ (5 x $5 \mathrm{~m}$ ), com base na metodologia empregada por Volpato, (1994). Estas subunidades foram implementadas no centro de 16 unidades amostrais de $250 \mathrm{~m}^{2}$ (10 x $\left.25 \mathrm{~m}\right)$, anteriormente locadas de forma permanente para o estudo da fitossociologia da comunidade arbórea adulta, com um distanciamento de $50 \mathrm{~m}$ entre si.

Os indivíduos mensurados receberam placas de alumínio com numeração crescente. Para mensurar a altura dos indivíduos que apresentaram até $2,0 \mathrm{~m}$, foi utilizada trena de bolso; quando necessário, estimou-se as demais com varas moduladas de $2,0 \mathrm{~m}$.

Os parâmetros fitossociológicos foram estimados utilizando-se o Microsoft EXCEL 2000. Os parâmetros avaliados foram: DR (Densidade Relativa); FR (Freqüência Relativa); RNC1 (Regeneração Natural na Classe de Altura 1); RNC2 (Regeneração Natural na Classe de Altura 2); RNC3 (Regeneração Natural na Classe de Altura 3); RNT (Regeneração Natural Total da População Amostrada).

As análises foram estabelecidas, com base no nível de inclusão, sendo mensurados os indivíduos que apresentaram CAP $\leq 15 \mathrm{~cm}$ e alturas correspondentes divididas em classes, sendo que a classe 1 contemplou indivíduos com $1,0 \leq \mathrm{h} \leq 2,0 \mathrm{~m}$; a classe 2, com indivíduos $2,0<\mathrm{h} \leq 3,0 \mathrm{~m}$; e a classe 3, indivíduos com $\mathrm{h}>3,0 \mathrm{~m}$.

Optou-se pela altura mínima de 1,0 m para a análise da regeneração de espécies arbóreas, pois, nesta altura, as espécies apresentam uma melhor definição da sua caracterização morfológica, permitindo uma identificação mais confiável.

A identificação taxonômica das espécies foi realizada no campo, sempre que possível. Quando necessário, foi coletado material botânico para identificação posterior no Herbário Dardáno de Andrade Lima - IPA (Empresa Pernambucana de Pesquisa Agropecuária), seguindo o sistema de classificação de Cronquist (1988).

Para cada espécie, foram estimados os parâmetros absolutos e relativos de freqüência e densidade, em cada classe de altura pré-estabelecida. Com base nesses parâmetros, estimou-se a regeneração natural por classe de altura, por meio das expressões que se seguem (VOLPATO, 1994):

$$
R N C_{i j}=\frac{D R_{i j}+F r_{i j}}{2}
$$

Em que: RNCij = estimativa da regeneração natural da i-ésima espécie na j-ésima classe de altura de planta, em percentagem; DRij = densidade relativa para a i-ésima espécie na j-ésima classe de altura de regeneração natural; FRij = freqüência relativa de i-ésima espécie, em percentagem, na j-ésima classe de regeneração natural.

Calculado o índice de regeneração por classe de altura para cada espécie, foi realizado o cálculo da estimativa da regeneração da população amostrada por espécie, utilizando-se da soma dos índices de regeneração natural por classe de altura, conforme Volpato (1994): 


$$
R N T_{i}=\sum\left(R N C_{i j}\right) / 3
$$

Em que: $\mathrm{RNTi}$ = estimativa da regeneração natural total da população amostrada da i-ésima espécie; RNCij = estimativa da regeneração natural da i-ésima espécie na j-ésima classe de altura de planta.

Para analisar a diversidade de espécies, foi utilizado o Índice de Diversidade de Shannon Wiener (H’), citado por Felfili e Rezende (2003):

$$
H^{\prime}=-\sum_{i=1}^{S} \frac{n_{i}}{N} \ln \frac{n_{i}}{N}
$$

Em que: $\mathrm{S}=$ número de espécies amostradas; $\mathrm{ln}=$ logaritmo neperiano; $\mathrm{n}_{\mathrm{i}}=$ número de indivíduos da espécie i; $\mathrm{N}$ = número total de indivíduos amostrados.

\section{Suficiência Amostral}

Para análise da suficiência amostral, utilizou-se o procedimento REGRELRP (Regressão Linear com Resposta em Platô) do Sistema para Análise Estatística e Genética - SAEG v.5.0, que já foi utilizado para a mesma finalidade por Costa-Neto (1990), Volpato (1994), Nappo et al. (1999), Barreira (1999), Gama et al. (2001), entre outros autores. A regressão linear com resposta em platô foi desenvolvida para análise de modelos matemáticos descontínuos, apresentando uma parte linear crescente e outra se sucedendo em platô.

O gráfico foi gerado por meio do "software Microsoft EXCEL for Windows ${ }^{\mathrm{TM}}$ 2000", que considera o número de pontos mínimos a ser amostrado e o ponto onde há a intersecção da parte linear crescente com a parte em forma de platô.

\section{RESULTADOS E DISCUSSÃO}

Por meio do estudo da suficiência amostral da análise de regressão com resposta em platô, verificouse que 16 parcelas $\left(400 \mathrm{~m}^{2}\right)$ foram suficientes para representar a composição florística da regeneração da Mata das Galinhas (Figura 2). De acordo com Ferreira (1988), a utilização desta metodologia é de extrema facilidade para a avaliação de amostragens florísticas, uma vez que minimiza o erro de estimação por meio dos mínimos quadrados e permite a retirada da subjetividade observada em outros métodos.

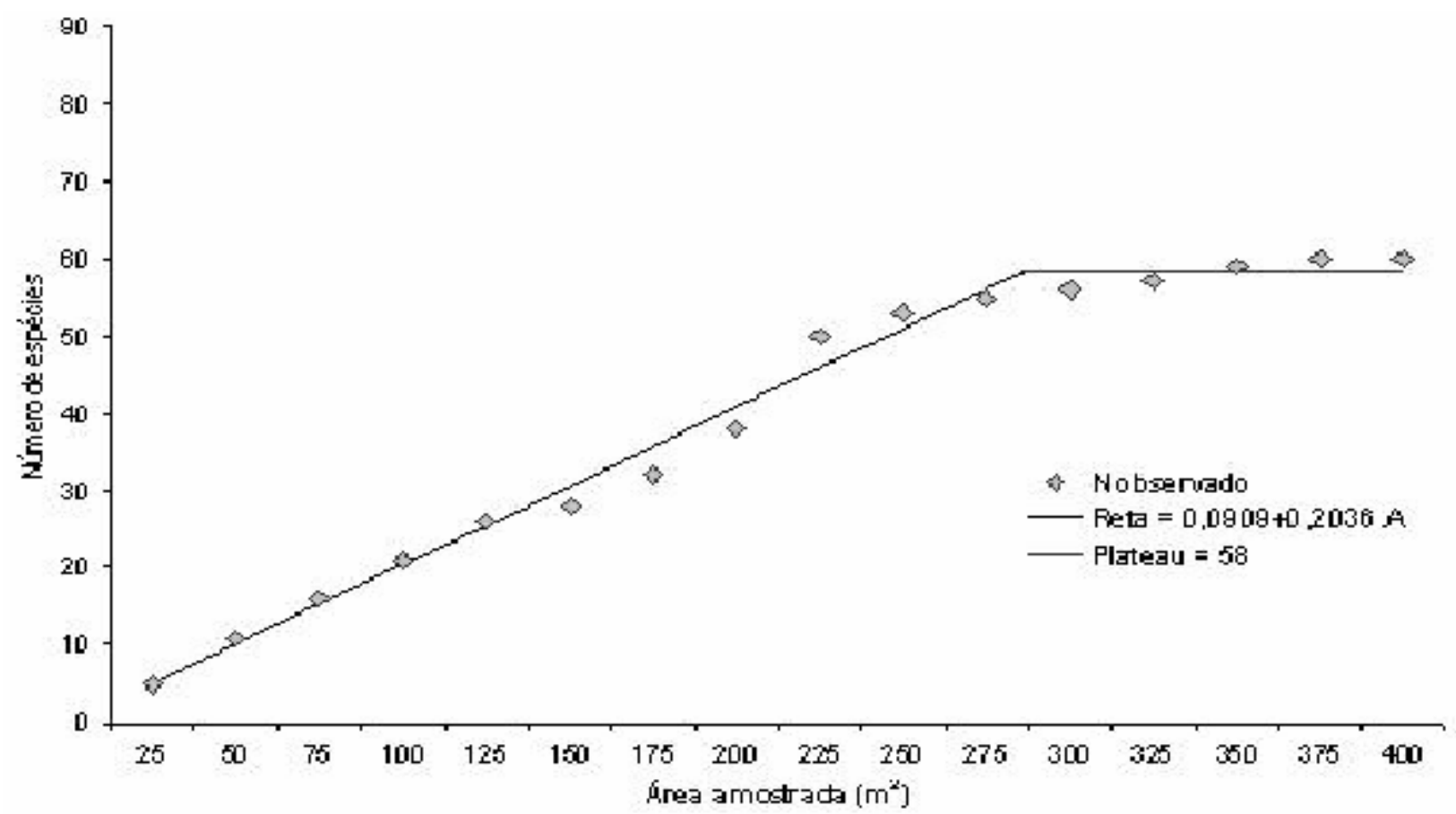

FIGURA 2: Determinação da suficiência amostral, utilizando-se o procedimento REGRELRP do SAEG, para o estudo da regeneração natural, no fragmento Mata das Galinhas, em Catende, PE.

FIGURE 2: Determination of samply sufficient, utilizing the REGRELRP method of SAEG, for natural regeneratios study's, Mata das Galinhas fragment, Catende’s city, PE. 
No fragmento Mata das Galinhas, foram amostrados 194 indivíduos vivos, pertencentes a 31 famílias botânicas, 48 gêneros e 60 espécies arbóreas (42 espécies foram identificadas em nível de espécie, 4 em nível de gênero, 2 em nível de família e 2 indeterminadas). As famílias que apresentaram maior destaque em número de espécies foram Mimosaceae, com 6 espécies; Moraceae, Lecythidaceae, Sapindaceae, Lauraceae, Meliaceae, Myrtaceae, Annonaceae e Flacourtiaceae, com 3 espécies cada; e Anacardiaceae, Burseraceae, Caesalpiniaceae e Melastomataceae com 2; as quais conjuntamente representaram 53,6\% da regeneração natural.

As dez espécies de maiores valores de regeneração natural total da população amostrada (Tabela 1), em ordem decrescente, foram as seguintes: Brosimum discolor (9,98\%), Protium heptaphyllum (9,19\%), Eschweilera ovata (8,01\%), Thyrsodium spruceanum (7,0\%), Dialium guianense (5,44\%), Erythroxylum squamatum (3,32\%), Cupania revoluta (3,16\%), Sorocea hilarii (2,66\%), Cordia nodosa (2,52\%) e Tapirira guianensis (2,50\%), representando 53,78\% da população amostrada.

TABELA 1: Estimativa da Regeneração Natural Total da População Amostrada (RNT) por classe de altura nas subunidades amostrais do fragmento Mata das Galinhas, em Catende, PE.

TABLE 1: Estimate of total natural regeneration of the samples population (RNT) height class in samples Mata das Galinhas fragment, Catende’s city, PE.

\begin{tabular}{|c|c|c|c|c|c|c|c|c|c|c|}
\hline Nome Científico & $\begin{array}{l}\text { DR1 } \\
(\%)\end{array}$ & $\begin{array}{l}\text { FR1 } \\
(\%)\end{array}$ & $\begin{array}{c}\text { RNC1 } \\
(\%)\end{array}$ & \begin{tabular}{|l|} 
DR2 \\
$(\%)$
\end{tabular} & $\begin{array}{l}\text { FR2 } \\
(\%)\end{array}$ & \begin{tabular}{|c|} 
RNC2 \\
$(\%)$ \\
\end{tabular} & \begin{tabular}{|l|} 
DR3 \\
(\%)
\end{tabular} & $\begin{array}{l}\text { FR3 } \\
(\%)\end{array}$ & \begin{tabular}{|c|} 
RNC3 \\
$(\%)$ \\
\end{tabular} & $\begin{array}{c}\text { RNT } \\
(\%)\end{array}$ \\
\hline$\overline{\text { Brosimum discolor Schott }}$ & 8,45 & 10,71 & 9,58 & 12,50 & 12,90 & 12,70 & 8,79 & 6,49 & 7,64 & 9,98 \\
\hline Protium heptaphyllum (Aubl.) Marchand & 9,86 & 10,71 & 10,29 & 9,38 & 9,68 & 9,53 & 7,69 & 7,79 & 7,74 & 9,19 \\
\hline Eschweilera ovata (Cambess.) Miers & 12,68 & 8,93 & 10,80 & 3,13 & 3,23 & 3,18 & 10,99 & 9,09 & 10,04 & 8,01 \\
\hline Thyrsodium spruceanum Benth. & 8,45 & 5,36 & 6,90 & 6,25 & 6,45 & 6,35 & 7,69 & 7,79 & 7,74 & 7,00 \\
\hline Dialium guianense (Aubl.) Sandv & 2,82 & 3,57 & 3,19 & 9,38 & 9,68 & 9,53 & 3,30 & 3,90 & 3,60 & 5,44 \\
\hline m squamatum & 0,00 & 0,00 & 0,00 & 6,25 & 6,45 & 6,35 & 3,30 & 3,90 & 3,60 & 3,32 \\
\hline oluta Rolfe & 1,41 & 1,79 & 1,60 & 3,13 & 3,23 & 3,18 & 5,49 & 3,90 & 4,70 & 3,16 \\
\hline a hilarii Gaudich. & 4,23 & 5,36 & 4,79 & 3,13 & 3,23 & 3,18 & 0,00 & 0,00 & 0,00 & 2,66 \\
\hline Cordia nodosa Lam. & 2,82 & 3,57 & 3,19 & 3,13 & 3,23 & 3,18 & 1,10 & 1,30 & 1,20 & 2,52 \\
\hline Гар & 4,23 & 3,57 & 3,90 & 0,00 & 0,00 & 0,00 & 3,30 & 3,90 & 3,60 & 2,50 \\
\hline yylla $\mathrm{N}$ & 4,23 & 3,57 & 3,90 & 3,13 & 3,23 & 3,18 & 0,00 & 0,00 & 0,00 & 2,36 \\
\hline Сирс & 1,41 & 1,79 & 1,60 & 0,00 & 0,00 & 0,00 & 6,59 & 3,90 & 5,24 & 2,28 \\
\hline Micon & 1,41 & 1,79 & 1,60 & 3,13 & 3,23 & 3,18 & 1,10 & 1,30 & 1,20 & 1,99 \\
\hline ndl.) & 2,82 & 3,57 & 3,19 & 0,00 & 0,00 & 0,00 & 2,20 & 2,60 & 2,40 & 1,86 \\
\hline Rheedia ga & 0,00 & 0,00 & 0,00 & 3,13 & 3,23 & 3,18 & 2,20 & 60 & 2,40 & 1,86 \\
\hline Ocotea glo & 2,82 & 1,79 & 2,30 & 3,13 & 3,23 & 3,18 & 0,00 & 0,00 & 0,00 & 1,83 \\
\hline & 4,23 & 3,57 & 3,90 & 0,00 & 0,00 & 0,00 & 1,10 & 1,30 & 1,20 & 1,70 \\
\hline & 0,00 & 0,00 & 0,00 & 6,25 & 3,23 & 4,74 & 0,00 & 0,00 & 0,00 & 1,58 \\
\hline & 0,00 & 0,00 & 0,00 & 3,13 & 3,23 & 3,18 & 1,10 & 1,30 & 1,20 & 1,46 \\
\hline Myrc & 0,00 & 0,00 & 0,00 & 3,13 & 3,23 & 3,18 & 1,10 & 1,30 & 1,20 & 1,46 \\
\hline $\begin{array}{l}\text { Allophylus edulis (A. St.-Hil., Cambess. } \\
\text { \& A. Juss.) Radlk. }\end{array}$ & 1,41 & 1,79 & 1,60 & 0,00 & 0,00 & 0,00 & 2,20 & 2,60 & 2,40 & 1,33 \\
\hline $\mathrm{DC}$ & 1,41 & 1,79 & 1,60 & 0,00 & 0,00 & 0,00 & 2,20 & 2,60 & 2,40 & 1,33 \\
\hline Nectal & 1,41 & 1,79 & 1,60 & 0,00 & 0,00 & 0,00 & 2,20 & 2,60 & 2,40 & 1,33 \\
\hline carpa D. Berg. & 0,00 & 0,00 & 0,00 & 3,13 & 3,23 & 3,18 & 0,00 & 0,00 & 0,00 & 1,06 \\
\hline & 0,00 & 0,00 & 0,00 & 3,13 & 3,23 & 3,18 & 0,00 & 0,00 & 0,00 & 1,06 \\
\hline cilipes (K. Schum.) A. & 0,00 & 0,00 & 0,00 & 3,13 & 3,23 & 3,18 & 0,00 & 0,00 & 0,00 & 1,06 \\
\hline Guatteria pogonopus Mart. & 0,00 & 0,00 & 0,00 & 3,13 & 3,23 & 3,18 & 0,00 & 0,00 & 0,00 & 1,06 \\
\hline & 0,0 & 0,00 & 0,00 & 3,13 & 3,23 & 3,18 & 0,00 & 0,00 & 0,00 & 1,06 \\
\hline & 0,0 & 0,00 & 0,00 & 3,13 & 3,23 & 3,18 & 0,00 & 0,00 & 0,00 & 1,06 \\
\hline Hime & 1,41 & 1,79 & 1,60 & 0,00 & 0,00 & 0,00 & 1,10 & 1,30 & 1,20 & 0,93 \\
\hline & & & & & & & & & & \\
\hline Plathymenia foliolosa Benth. & 1,41 & 1,79 & 1,60 & 0,00 & 0,00 & 0,00 & 1,10 & 1,30 & 1,20 & 0,93 \\
\hline
\end{tabular}


TABELA 1: Continuação ...

TABLE 1: Continued ...

\begin{tabular}{|c|c|c|c|c|c|c|c|c|c|c|}
\hline Jome Científico & $\begin{array}{l}\text { DR1 } \\
(\%)\end{array}$ & $\begin{array}{l}\text { FR1 } \\
(\%)\end{array}$ & $\begin{array}{c}\text { RNC1 } \\
(\%)\end{array}$ & $\begin{array}{l}\text { DR2 } \\
(\%)\end{array}$ & $\begin{array}{l}\text { FR2 } \\
(\%)\end{array}$ & \begin{tabular}{|c|} 
RNC2 \\
$(\%)$ \\
\end{tabular} & $\begin{array}{l}\text { DR3 } \\
(\%)\end{array}$ & $\begin{array}{l}\text { FR3 } \\
(\%)\end{array}$ & \begin{tabular}{|c|} 
RNC3 \\
$(\%)$
\end{tabular} & $\begin{array}{l}\text { RNT } \\
(\%)\end{array}$ \\
\hline outeria grandiflora (A. DC.) Baehni & 1,41 & 1,79 & 1,60 & 0,00 & 0,00 & 0,00 & 1,10 & 1,30 & 1,20 & 0,93 \\
\hline ylopia frutescens Aubl. & 41 & 1,79 & 1,60 & 0,00 & 0,00 & 0,00 & 1,10 & 30 & 1,20 & ,93 \\
\hline Taytenus distichophylla Mart. & 0,00 & 0,00 & 0,00 & 0,00 & 0,00 & 0,00 & 2,20 & 2,60 & 2,40 & 0,80 \\
\hline richilia silvatica C. DC. & 0,00 & 0,00 & 0,00 & 0,00 & 0,00 & 0,00 & 2,20 & 2,60 & 2,40 & 0,80 \\
\hline Casec & 2,82 & 1,79 & 2,30 & 0,00 & 0,00 & 0,00 & 0,00 & 0,00 & 0,00 & 0,77 \\
\hline uar & 2,82 & 1,79 & 2,30 & 0,00 & 0,00 & 0,00 & 0,00 & 0,00 & 0,00 & 0,77 \\
\hline аурс & 2,82 & 1,79 & 2,30 & 0,00 & 0,00 & 0,00 & 0,00 & 0,00 & 0,00 & 0,77 \\
\hline opai & 0,00 & 0,00 & 0,00 & 0,00 & 0,00 & 0,00 & 2,20 & 1,30 & 1,75 & 0,58 \\
\hline actr & 1,41 & 1,79 & 1,60 & 0,00 & 0,00 & 0,00 & 0,00 & 0,00 & 0,00 & 0,53 \\
\hline ase & 1,41 & 1,79 & 1,60 & 0,00 & 0,00 & 0,00 & 0,00 & 0,00 & 0,00 & 0,53 \\
\hline ase & 1,41 & 1,79 & 1,60 & 0,00 & 0,00 & 0,00 & 0,00 & 0,00 & 0,00 & 0,53 \\
\hline & 1,41 & 1,79 & 1,60 & 0,00 & 0,00 & 0,00 & 0,00 & 0,00 & 0,00 & 0,53 \\
\hline $\operatorname{Iim}$ & 1,41 & 1,79 & 1,60 & 0,00 & 0,00 & 0,00 & 0,00 & 0,00 & 0,00 & 0,53 \\
\hline toni (Aubl.) Maguire, & 1,41 & 1,79 & 1,60 & 0,00 & 0,00 & 0,00 & 0,00 & 0,00 & 0,00 & 0,53 \\
\hline mulcherrimum (Willd ) & 1,41 & 1,79 & 1,60 & 00 & 0,00 & 0,00 & 0,00 & 0 & 0,00 & 3 \\
\hline uphorbi & 0,00 & 0,00 & 0,00 & 0,00 & 0,00 & 0,00 & 1,10 & 1,30 & 1,20 & 0,40 \\
\hline nde & 0,0 & 0,00 & 0,0 & 0,00 & 0,00 & 0,00 & 1,10 & 1,30 & 1,20 & 0,40 \\
\hline ndet & 0,0 & 0,00 & 0,00 & 0,00 & 0,00 & 0,00 & 1,10 & 1,30 & 1,20 & 0,40 \\
\hline $\operatorname{lgc}$ & 0,0 & 0,00 & 0,00 & 0,00 & 0,00 & 0,00 & 1,10 & 1,30 & 1,20 & 0,40 \\
\hline ec) & 0,0 & 0,00 & 0,00 & 0,00 & 0,00 & 0,00 & 1,10 & 1,30 & 1,20 & 0,40 \\
\hline$I a c$ & 0,00 & 0,00 & 0,00 & 0,00 & 0,00 & 0,00 & 1,10 & 1,30 & 1,20 & 0,40 \\
\hline$i c 0$ & & 0,00 & $0,($ & 0,00 & 0,00 & 0,00 & 1,10 & 1,30 & 1,20 & 0,40 \\
\hline $\begin{array}{l}\text { Ourate } \\
\text { Baill. }\end{array}$ & 0,00 & 0,00 & 0,00 & 0,00 & 0,00 & 0,00 & 1,10 & 1,30 & 1,20 & 0,40 \\
\hline Pogonophora schomburgkiana Miers ex & 0,00 & 0,00 & 0,00 & 0,00 & 0,00 & 0,00 & 1,10 & 1,30 & 1,20 & 0,40 \\
\hline & 0,00 & 0,00 & 0,00 & 0,00 & 0,00 & 0,00 & 1,10 & 1,30 & 1,20 & 0,40 \\
\hline Psychotria sessilis Vell. & 0,00 & 0,00 & 0,00 & 0,00 & 0,00 & 0,00 & 1,10 & 1,30 & 1,20 & 0,40 \\
\hline Quiina sp. & 0,00 & 0,00 & 0,00 & 0,00 & 0,00 & 0,00 & 1,10 & 1,30 & 1,20 & 0,40 \\
\hline Rollinia sp. & 0,00 & 0,00 & 0,00 & 0,00 & 0,00 & 0,00 & 1,10 & 1,30 & 1,20 & 0,40 \\
\hline Siparuna guianensis Aubl. & 0,00 & 0,00 & 0,00 & 0,00 & 0,00 & 0,00 & 1,10 & 1,30 & 1,20 & 0,40 \\
\hline Total & 100 & 100 & 100 & 100 & 100 & 100 & 100 & 100 & 100 & 100 \\
\hline
\end{tabular}

Em que: DR= Densidade Relativa; FR = Freqüência Relativa; RNC1 = Regeneração Natural na Classe 1 de altura; RNC2 = Regeneração Natural na Classe 2 de altura; RNC3 = Regeneração Natural na Classe 3 de altura.

Utilizando o mesmo método para o estudo da regeneração natural em um fragmento de floresta atlântica no município do Cabo de Santo Agostinho, Zona da Mata Sul de Pernambuco, Silva Júnior (2004) encontrou, com maiores valores para regeneração natural (RN), as espécies Annona glabra (9,16\%), Brosimum conduru (5,90\%), Paypayrola blanchetuana (5,88\%), Protium giganteum (5,20\%), Eschweilera ovata (5,06\%) e Brosimum discolor (5,03\%), Pouteria grandiflora (4,32\%), Manilkara salzmanii (4,27\%), Nectandra cuspidata (3,95\%) e Siparuna guianensis (3,93\%).

Para este trabalho, as espécies que se destacaram com maior número de indivíduos foram, respectivamente, Eschweilera ovata (20), Brosimum discolor (18) e Protium heptaphyllum (17) (Figura 3). 


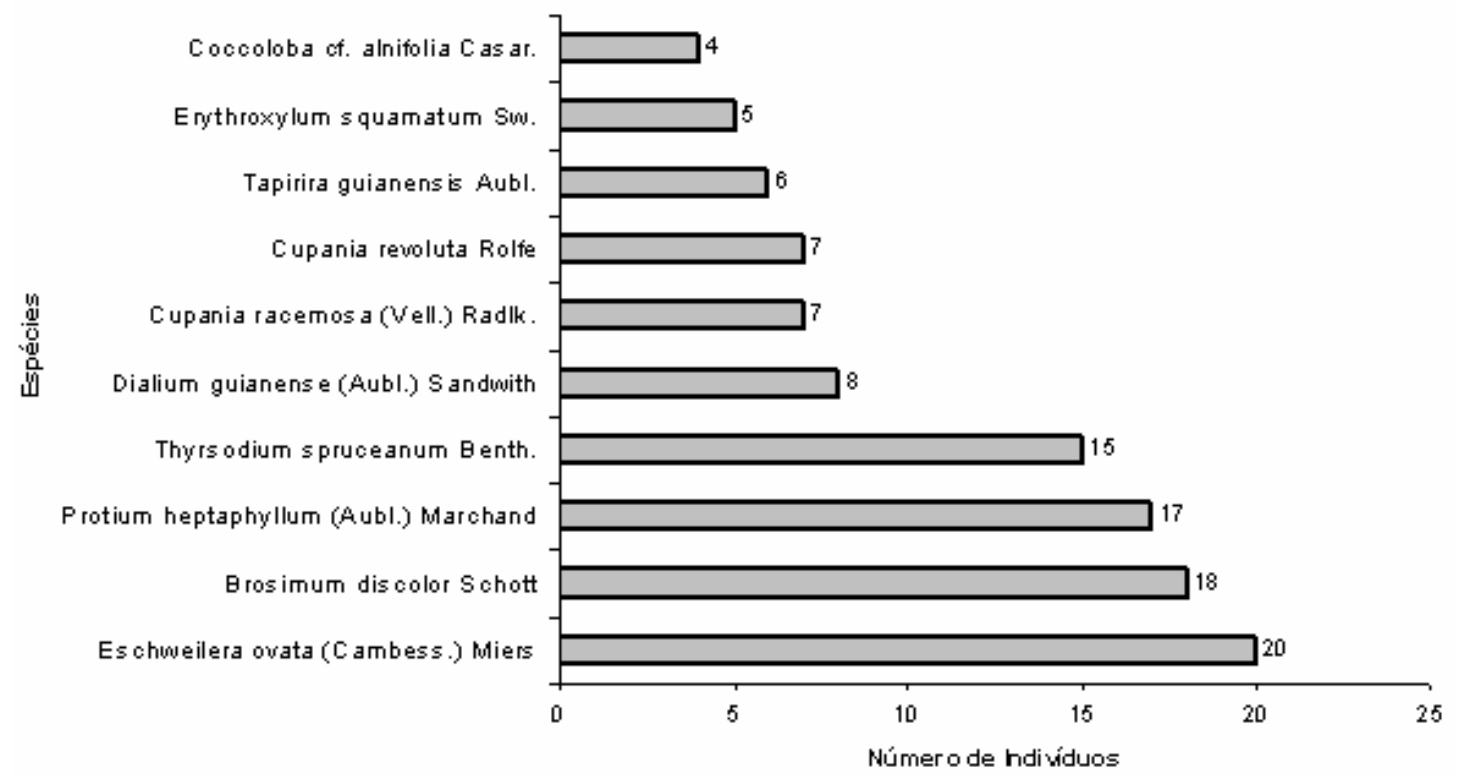

FIGURA 3: Relação entre espécies e número de indivíduos nas 16 subparcelas no fragmento Mata das Galinhas, em Catende, PE.

FIGURE 3: Relation between species and numbers of individuals in 16 subunits in the Mata das Galinhas fragment, Catende's city, PE.

Guimarães (2005), estudando a estrutura e a fitossociologia das espécies arbóreas adultas no mesmo fragmento de estudo (Mata das Galinhas - Catende/PE), observou que a espécie Brosimum discolor também se destacou para o fragmento sendo a de maior valor de importância para a área, seguida das espécies Protium heptaphyllum, Eschweilera ovata, Thyrsodium spruceanum e Tapirira guianensis. O resultado por ele encontrado corrobora com os dados obtidos neste trabalho.

Dentre as 60 espécies amostradas, as que apresentaram, para Regeneração Natural na Classe 1 (RNC1), maiores percentuais foram Eschweilera ovata (10,8\%), Protium heptaphyllum (10,2\%) e Brosimum discolor (9,5\%) (Figura 4).

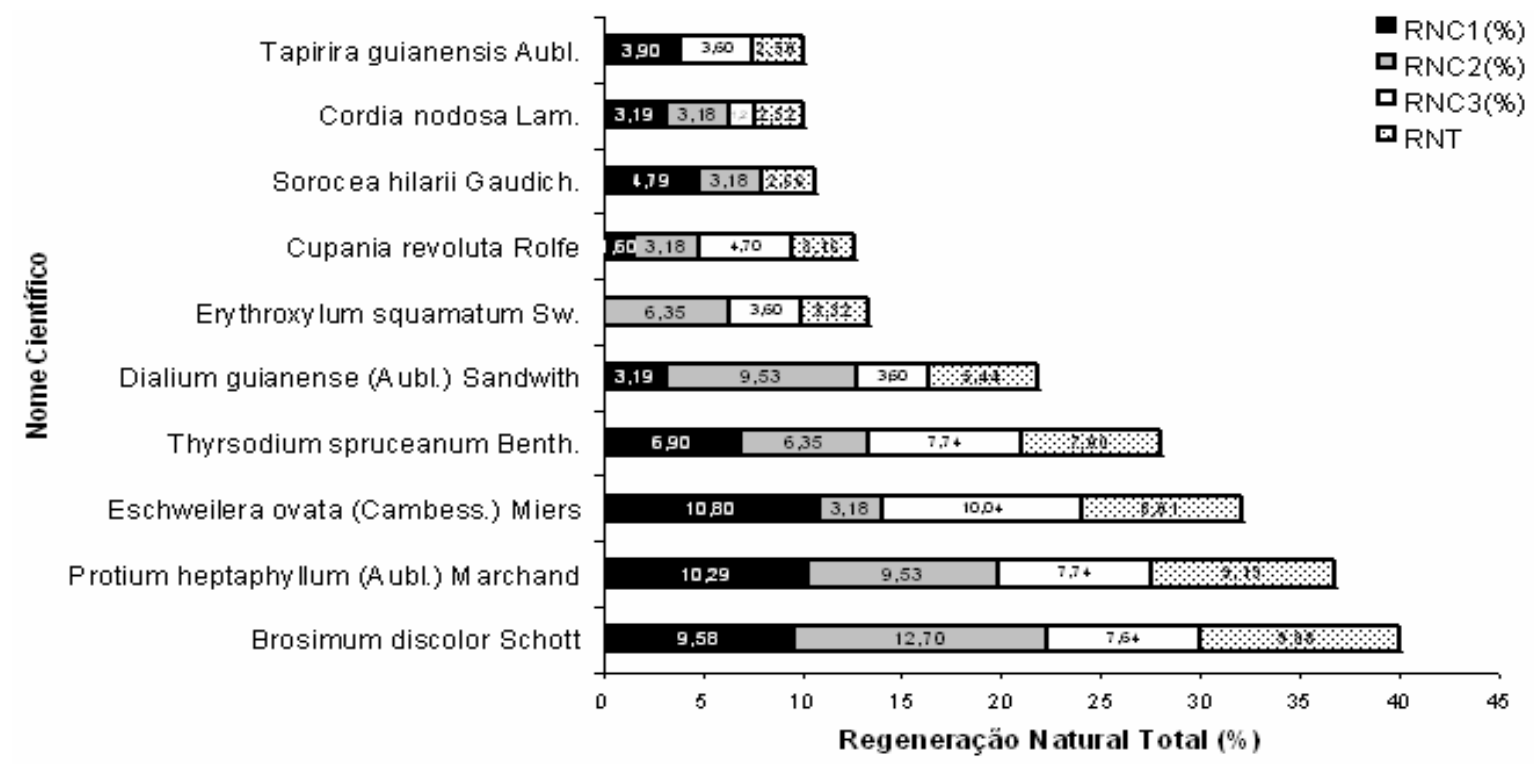

FIGURA 4: Relação das 10 espécies que apresentam valores mais altos de regeneração natural total da população amostrada (RNT), expressas em percentagem, amostradas no fragmento Mata das Galinhas, em Catende, PE.

FIGURE 4: Relation of 10 species that show elevated values of total natural regeneration of the samples population (RNT) in percentage, in the Mata das Galinhas fragment, Catende’s city, PE. 
Para Regeneração Natural na Classe 2 (RNC2), as que se destacaram foram Brosimum discolor (12,7\%), Protium heptaphyllum (9,53\%), Dialium guianense (9,53\%) e Thyrsodium spruceanum (6,35\%).

Para Regeneração Natural na Classe 3 (RNC3), as espécies que apresentaram maiores valores foram Eschweilera ovata (10,04\%), Protium heptaphyllum (7,74\%), Thyrsodium spruceanum (7,74\%) e Brosimum discolor (7,64\%).

Observou-se que 34 espécies estão presentes em apenas uma classe de altura. Embora possuam uma representatividade menor e apresentem mudanças nos valores de regeneração natural entre as classes de tamanho, possivelmente poder-se-á evidenciar um futuro ingresso de tais espécies no estrato arbóreo. A ausência dessas populações na menor classe de altura pode estar ligada a estratégias de ocupação de espaço, com dependência de clareiras para se estabelecerem. Além disso, observou-se a presença de 18 espécies em duas classes, fornecendo subsídios para a estimativa de que estejam presentes na área em um estágio de sucessão mais avançado. Apenas 8 estão presentes em todas as classes de altura.

As espécies que ocorrem na três classes de altura de regeneração natural na comunidade são aquelas que teoricamente possuem um maior potencial de estabelecimento na floresta e que deverão estar presentes no futuro dossel, desde que seja realizado seu acompanhamento durante o crescimento, observando-se suas características sucessionais. De acordo com Citadini-Zanette (1995), as espécies que ocorrem em todas as classes de altura, de maneira geral, são aquelas que estariam melhor representadas na composição futura da floresta, ou seja, aquelas que estão mais aptas a se estabelecerem na biocenose.

Por outro lado, sabe-se que a ocorrência de distúrbios naturais ou antrópicos cria habitats altamente heterogêneos no ambiente da floresta, os quais, por sua vez, proporcionam o recrutamento de diferentes espécies de plantas, mostrando diferentes paisagens de regeneração (UHL e MURPHY 1981; VITOUSEK e DENSLOW, 1986).

Dessa forma, os resultados aqui apresentados não podem garantir, mas permitem presumir que as espécies encontradas com uma alta capacidade de regeneração realmente estejam presentes na futura floresta. Entretanto, ressalta-se que é importante um acompanhamento da área estudada, associado ao monitoramento do crescimento das espécies amostradas, de modo a permitir um real entendimento sobre a dinâmica de reposição do dossel.

No entanto, foi constatado, para aproximadamente 60\% das espécies observadas, densidades relativas inferiores a 1 em pelo menos uma classe de altura. Segundo Kageyama e Gandara (1993), estas espécies devem ser preservadas na área, uma vez que não apresentam facilidade para se instalar.

Dentre as 60 espécies amostradas, as que apresentaram maior freqüência relativa nas classes de altura foram Brosimum discolor e Protium heptaphyllum, nas classes 1 e 2 de altura, e Eschweilera ovata, na classe de altura 3.

As espécies Brosimum discolor (10\%), Protium heptaphyllum (9,2\%), Eschweilera ovata (8,0\%) e Thyrsodium spruceanum (7,0\%) foram consideradas as mais representativas na regeneração natural, presumindo-se, assim, que ambas provavelmente serão bem sucedidas no futuro da floresta.

Das 60 espécies, 31 obtiveram valores de RNT inferiores a 1,0\%, o que indica, a partir de um determinado tempo, que elas podem passar a ter um maior grau de dificuldade em se regenerar ou que são espécies tardias que estão agora entrando no ecossistema.

No que se refere à distribuição das espécies nas classes de altura, as que se destacaram por estarem bem representadas nas três classes de Regeneração natural amostrada foram Brosimum discolor e Protium heptaphyllum. Isto pode estar relacionado ao fato de a taxa de crescimento ser predominante sobre a taxa de mortalidade. Segundo Jardim (1990), estas espécies são as principais responsáveis pela manutenção da estrutura e fisionomia da floresta.

A espécie Eschweilera ovata (Camb.) Miers está bem representada nas três classes, embora apresente um menor desempenho na classe 2, com poucas variações nos valores de regeneração natural entre as classes de tamanho, sendo, portanto, uma espécie que poderá evidenciar um futuro ingresso no estrato arbóreo.

Algumas espécies destacaram-se consideravelmente em duas classes de altura, dentre as quais estão Thyrsodium spruceanum e Dialium guianense, fornecendo subsídios para a estimativa de que a espécie esteja 
presente na área em um estádio de sucessão mais avançado.

De acordo com os dados obtidos neste trabalho, é factível afirmar que a formação vegetacional da área estudada no município de Catende, Pernambuco, corresponde à Floresta Ombrófila Densa, o que corrobora com o trabalho desenvolvido por Ranta et al. (1998) nas proximidades do município. Entretanto, discorda da afirmação apresentada pelo CONDEPE (1987), que a classificou como Floresta Estacional Semidecidual.

O resultado do índice de diversidade de Shannon-Wiener (H’) encontrado para o fragmento estudado foi de 3,57 nats/ind.. Tal valor confirma a importância da conservação dos fragmentos para a região, considerando a alta biodiversidade em uma área com poucos estudos realizados, o que exige ações prioritárias e urgentes de conservação. Os fatores que provavelmente contribuíram para a considerável diversidade são, basicamente, a existência de fontes de regeneração na floresta, brotação de cepas e banco de sementes, além da presença de animais dispersores de sementes.

\section{CONCLUSÕES}

A Brosimum discolor é a espécie que obteve o melhor desempenho para a comunidade estudada, com adaptação mais eficiente às condições apresentadas pelo sítio quando comparada com as demais populações.

Foi constatado, na área de estudo, a ocorrência de Brosimum discolor, Cordia nodosa, Cupania revoluta, Dialium guianense, Eschweilera ovata, Miconia prasina, Protium heptaphyllum e Thyrsodium spruceanum, nas três classes de altura, espécies as quais, possivelmente, estarão presentes na floresta futura, devendo ser priorizadas para a recuperação de áreas degradadas na região.

\section{REFERÊNCIAS BIBLIOGRÁFICAS}

BARREIRA, S. Estudo da regeneração natural de cerrado como base para o manejo florestal. 1999. $113 \mathrm{f}$. Dissertação (Mestrado em Engenharia Florestal) - Universidade Federal de Lavras, Lavras, 1999.

BARREIRA, S.; SCOLFORO, J. R. S.; BOTELHO, S. A. et al. Estudo da estrutura da regeneração natural e da vegetação adulta de um cerrado senso stricto para fins de manejo florestal. Scientia Forestalis, Piracicaba, n. 61, p. 6478, 2002.

CITADINI-ZANETTE, V. Fitossociologia e aspectos dinâmicos de um remanescente da Mata Atlântica na microbacia do Rio Novo, Orleans, SC. 1995. 236 f. Tese (Doutorado em Ecologia e Recursos Naturais) Universidade Federal de São Carlos, São Carlos, 1995.

CONDEPE. Catende. Recife: CONDEPE, 1987. 62 p. (Monografias Municipais, 27).

COSTA-NETO, F. Subsídios técnicos para um plano de manejo sustentado em áreas de Cerrado. 1990. 142 f. Dissertação (Mestrado em Engenharia Florestal) - Universidade Federal de Viçosa, Viçosa, 1990.

CRONQUIST, A. The evolution and classification of flowering plants. $2^{\circ}$. ed., New York: The New York Botanical Garden, 1988, 555 p.

EMBRAPA (Empresa Brasileira de pesquisa Agropecuária). Sistema Brasileiro de Classificação dos Solos. 2005. Disponível em: <http: www.cnps.embrapa.br/sibcs/>, acessado em 02/07/05.

FELFILI, J. M.; REZENDE, R. P. Conceitos e métodos em fitossociologia. Brasília: UNB, 2003. 68 p. (Comunicações Técnicas Florestais - ISSN, v.5, n.1).

FERREIRA, R. L. C. Análise estrutural da vegetação da estação florestal de experimentação de Açu-RN, como subsídio básico para o manejo florestal. 1988. 90 f. Dissertação (Mestrado em Ciência Florestal) - Universidade Federal de Viçosa, Viçosa, 1988.

FINOL, U. H. Nuevos parametros a considerarse en el analisis estrutural de las selvas virgenes tropicales. Revista Forestal Venezolana, Mérida, v. 18, n. 12, p. 29-42, 1971.

GAMA, J. R. V.; BOTELHO, S. A.; BENTES-GAMA, M. de M. et al. Tamanho de parcela e suficiência amostral para estudo da regeneração natural em floresta de várzea na Amazônia. Revista Cerne, Lavras, v. 7, n. 2, p. 001-011, 2001.

GUIMARÃES, F. J. P. Avaliação da estrutura de um fragmento florestal no município de Catende/PE. 2005. $60 \mathrm{f}$. Dissertação (Mestrado em Ciências Florestais) - Universidade Federal Rural de Pernambuco, Recife, 2005.

JARDIM, F. C. S. Mortalidade e crescimento na floresta equatorial de terra firme. Belém: Museu Paraense Emílio 
Goeldi, Série Botânica, v. 6, n. 2, p. 227-234, 1990.

KAGEYAMA, P. Y.; GANDARA, F. B. Dinâmica de populações de espécies arbóreas para o manejo e a conservação. In: SIMPÓSIO DE ECOSSISTEMAS DA COSTA RASILEIRA, 3., 1993, São Paulo. Anais ... São Paulo: ACIESP, 1993. p. 1-9.

NAPPO, M. E.; FONTES, M. A. L.; OLIVEIRA-FILHO, A. T. de. Suficiência amostral e análise do tamanho de parcelas para o estudo da regeneração natural do sub-bosque de povoamentos homogêneos de Mimosa scabrella Benth., em área minerada, em Poços de Caldas-MG. Revista Árvore, Viçosa, v. 23, n. 4, p. 443-453, 1999.

RANTA, P.; BLOM, T.; NIEMELÃ, J. et al. The fragmented Atlantic rain forest of Brazil: size, shape and distribution of forest fragments. Biodiversity and Conservation, New York, v. 7, p. 385-403, 1998.

RONDON NETO, R. M.; BOTELHO, S. A.; FONTESA, M. A. L. et al. Estrutura e Composição Florística da Comunidade Arbustivo-Arbórea de uma Clareira de Origem Antrópica, em uma Floresta Estacional Semidecídua Montana, Lavras-MG, Brasil. Revista Cerne, Lavras, v. 6, n. 2, p. 79-94, 2000.

SANTANA, C. A. de A.; LIMA, C. C. D. de; MAGALHÃES, L. M. S. Estrutura horizontal e composição florística de três fragmentos secundários na cidade do Rio de Janeiro. Acta Scientiarum Biological Sciences, Maringá, v. 26, n. 4, p. 443-451, 2004.

SILVA JÚNIOR, J. F. Estudo fitossociológico em um remanescente de floresta atlântica visando dinâmica de espécies florestais arbóreas no município do Cabo de Santo Agostinho, PE. 2004. 84 f. Dissertação (Mestrado em Ciências Florestais) - Universidade Federal Rural de Pernambuco, Recife, 2004.

SWAINE, M. D.; HALL, J. B. The Mosaic theory of forest regeneration and the determination of forest composition in Ghana. Journal of Tropical Ecology, Cambridge, v. 4, p. 253-269, 1988.

VOLPATO, M. M. L. Regeneração natural em uma floresta secundária no domínio de Mata Atlântica: uma análise fitossociológica. 1994. 123 f. Dissertação (Mestrado em Ciência Florestal) - Universidade Federal de Viçosa, Viçosa, 1994. 